\title{
Masters Spring Academy 2011 in Bremen
}

\author{
Miriam Düsterhöft und André Völker
}

Fukushima ist ein schwarzer Schwan. Die Ereignisse in Japan gehören zu jenen Vorkommnissen, die eigentlich nicht passieren dürften und dennoch passiert sind. Mit dem Verweis auf die politikwissenschaftliche Herausforderung durch höchst unwahrscheinliche Ereignisse leitete Friedbert Rüb von der HU Berlin einen Kurs zum Multiple Streams Ansatz und damit die mittlerweile vierte Masters Spring Academy on Policy Analysis und German Policy Studies Conferences ein.

Vom 31. März bis zum 2. April 2011 diskutierten Masterstudierende, Nachwuchswissenschaftler und etablierte Vertreter der verschiedenen Perspektiven der Policy Analyse in Bremen aktuelle Fragestellungen und neue Trends. Im Rahmen von Workshops der Online-Zeitschrift German Policy Studies konnten die Teilnehmenden nicht nur mit Autoren über aktuelle und geplante Fachaufsätze diskutieren, sondern auch eigene Beiträge einbringen und sich an eventuellen Publikationsvorhaben beteiligen. Die Veranstaltung konnte einen Überblick über die wichtigsten aktuellen Entwicklungen in den theoretischen Perspektiven zur Policy Analyse geben und diese mit aktuellen Debatten insbesondere zur Umweltpolitik verbinden.

Nach der Begrüßung und Einführung durch Nils Bandelow von der TU Braunschweig und Herbert Obinger von der Universität Bremen sprach Rüb über den Multiple-StreamsAnsatz. Rüb entwickelte die These, dass sich die Welt in einem Umbruch befinde, gekennzeichnet von einem zunehmenden Wirklichkeits- und Wissenszerfall. Politische Entscheidungen sind dabei immer häufiger von einer zeitorientierten Reaktivität geprägt und weniger, wie früher, von einer zielorientierten Rationalität. Doch wie kann es politischen Akteuren in diesem relativ ungesteuerten Entscheidungsprozess dann noch gelingen, einzelne Sachverhalte gezielt auf die politische Agenda zu setzen? Das Geschick, so Rüb, läge dabei im gezielten Herausstellen einzelner (Problem-)Ströme und dem Ausnutzen bestimmter Zeitfenster. Die schwarzen Schwäne und damit die Ereignisse in Fukushima bilden solche Zeitfenster. Sie eröffnen die Möglichkeit zu wichtigen Entscheidungen. Es bleibt aber die starke Zufälligkeit des „Wie“ und „Wann“, Prognosen sind damit nur schwer möglich. Rüb weist aber auch darauf hin, dass das Multiple-Streams-Konzept hier die, zumindest in Bezug auf die Zeitstrukturen, klaren Regelungen vieler Institutionen übersieht.

Unvermeidbar im Kontext neuerer Ansätze der Policy-Forschung ist die Vetospielertheorie, in Bremen vorgestellt durch Reimut Zohlnhöfer aus Bamberg. Zohlnhöfer diskutierte Definitionen und Operationalisierungsmöglichkeiten der zentralen Variablen: Zahl der Vetospieler, Kongruenz der Vetospieler und Kohäsion innerhalb der Vetospieler. Er stellte die Hypothesen und die aktuellen Fachdebatten zu jüngeren Ent- wicklungen der Theorie vor. Besonderen Wert legt er auf die Klärung, dass George Tsebelis keine eigenen Aussage darüber macht, ob oder wann Reformen stattfinden, sondern lediglich darüber, wodurch Wandel nicht wahrscheinlich wird. In der nachfolgenden Debatte wurde deutlich, dass eine große Gruppe der Anwesenden die Konzepte der Vetospielertheorie eher qualitativ verwenden möchte. Der Ertrag bei einer formalen Anwendung der Theorie im Verhältnis zum Forschungsaufwand wurde kritisch diskutiert.

Der letzte Redner an diesem Tag war Nils Bandelow von der TU Braunschweig, der theoretische Grundlagen, aktuelle Entwicklungen und Herausforderungen für eigene Forschungen auf Grundlage des Advocacy Coalition Frameworks (ACF) nach Paul Sabatier vorstellte. Dieser analytische Rahmen soll langfristige Veränderungen erklären und dabei auch Policy-Lernen als mögliche Erklärung berücksichtigen. Wichtige Elemente des Ansatzes liefern aber auch Erklärungen für Policy-Stabilität: Hierzu gehören etwa die hierarchisch strukturierten Belief-Systeme. Insbesondere allgemeine Normen und Werte sind kaum veränderlich. Auch das zweite zentrale Element des Ansatzes, die Advocacy-Koalitionen, können eher Stabilität als Veränderungen erklären. Advocacy-Koalitionen werden als Zusammenschlüsse von Akteuren in Policy-Subsystemen gesehen. Sie basieren auf übereinstimmenden Beliefs, sind aber auch rationalistisch begründet, um die Durchsetzung gemeinsamer Interesen zu ermöglichen. Während in den ursprünglichen Versionen des Ansatzes vor allem externe Ereignisse und Lernen als zentrale Erklärungen für PolicyVeränderungen gesehen werden, ist inzwischen eine schrittweise Erweiterung zu beobachten. Bandelow sieht diese Erweiterungen überwiegend kritisch: Der Informationsgehalt der Hypothesen werde dadurch weiter reduziert und die analytische Klarheit nehme ab. Gleichzeitig ist aber die methodische Klarheit der jüngsten Studien auf Grundlage des ACF gestiegen. Der Ansatz wird offenbar zunehmend auch in europäischen Kontexten angewendet und scheint vor allem für umwelt- und technologiepolitische Policies geeignet zu sein.

Am zweiten Tag stellte zunächst Susanne Schmidt den akteurzentrierten Institutionalismus nach Renate Mayntz und Fritz W. Scharpf vor. Der Ansatz bedient sich zwar spieltheoretischer Heuristiken, geht aber nicht grundsätzlich davon aus, dass Akteure rational handeln. Institutionen werden definiert als Regeln und Normen, die den Akteuren bekannt sind. Sie bestimmen die Präferenzen der Akteure und machen ihr Handeln berechenbar. Der Ansatz verfolgt laut Schmidt aber nicht die Vorhersage von Ereignissen, sondern die Analyse vergangener Entscheidungen, um daraus eventuell Handlungsanweisungen für die Zukunft ableiten zu können. Probleme werden innerhalb des institutionellen Kontextes von 
Akteurskonstellationen und deren Interaktion gelöst. Hier zeigen sich die Elemente aus der Spieltheorie, denn Akteure werden als Spieler bezeichnet und handeln nach Strategieoptionen und Präferenzen. Welcher Art die Interaktionsformen sind ist abhängig von den jeweiligen institutionellen Regeln. Es kann durch einseitiges Handeln, Verhandlung, Mehrheitsentscheidung oder hierarchische Steuerung interagiert werden. Hieraus resultiert, dass verschiedene Interaktionsformen politische Problemlagen auch unterschiedlich gut lösen können. Jedoch spielt nicht nur die Form der Interaktion eine Rolle, sondern auch die Orientierung. Akteure können rational, kooperativ, altruistisch, kompetitiv oder feindlich handeln. Bei der Untersuchung der Handlungsoptionen ist wichtig, dass keine vollständige Erklärung möglich ist, aber vieles durch Institutionen geprägt wird. Schmidt stellte vor allem heraus, dass der Ansatz, obwohl von Renate Mayntz und Fritz W. Scharpf nicht weiterentwickelt, vielfältig genutzt und anderweitig weiterentwickelt wurde.

Auf diese Ausführungen folgte der Vortrag von Peter Starke, der den in Deutschland noch vergleichsweise wenig verbreiteten Punctuated-Equilibrium-Ansatz vorstellte. Der Begriff des Punctuated Equilibrium stammt aus der Evolutionstheorie und bezeichnet eine Entwicklung von Arten, die sprunghaft und nicht kontinuierlich verläuft. Der Ansatz hat den Anspruch gleichzeitig Kontinuität und Wandel erklären zu können. Änderungen von politischen Inhalten müssen in der Summe immer möglichst normalverteilt sein, deswegen müssen Veränderungen durch die Politik so verarbeitet werden, dass diese Normalverteilung immer wieder hergestellt werden kann. Genutzt wird bei der Untersuchung dieser Vorgänge die Wahrscheinlichkeitstheorie. Es wird außerdem von begrenzter Rationalität der Akteure und von einem politischen System, welches sich aus Makropolitik und einem Policy-Subsystem zusammensetzt, gesprochen. Das Verhältnis dieser beiden wird durch Institutionen bestimmt und es ergeben sich zwei Formen von Dynamiken. Die erste Form ist das „,negative Feedback“, welches innerhalb des Policy-Subsystems stattfindet und versucht, entstandene negative Aspekte abzuschwächen. Die zweite Form ist das „positive Feedback“, welches einen Prozess innerhalb der Makropolitik bezeichnet, bei dem eine kleine Veränderung zu einem großen Wandel führt. Dies geschieht durch ein sogenanntes „policy image“, welches Emotionen und Informationen, die den Prozess beschleunigen, bezeichnet. Diese Veränderungen sind sprunghaft. Kontrovers wurde diskutiert, inwiefern der Ausgangsbefund des Ansatzes den bekannten Prozessen und Ergebnissen in der Politik gerecht wird.

Höhepunkt der Veranstaltung war ein Vortrag des Politikwissenschaftlers und Bremer Senators für Umwelt, Bau, Verkehr und Europa, Reinhard Loske, der den theoriegeleiteten Kontext mit dem Politikfeld der Umweltpolitik verband. Er gab zunächst einen kleinen historischen Abriss über die Entwicklung und die Institutionalisierung der Umweltpolitik in Deutschland in den letzten 40 Jahren. Er skizzierte dabei vor allem, wie die Umweltpolitik immer mehr an Bedeutung gewann und sich immer stärker der Vor- statt Nachsorge verpflichtete. Gerade in den letzten Jahren hat das Thema Nachhaltigkeit in Deutschland und der Welt enorm an Bedeutung gewonnen. Aber immer noch sehen viele Unternehmen Umweltschutz eher als „burden“, denn als Chance. Loske betonte, dass der Umweltschutz einen internationalen Rahmen mit entsprechenden gültigen Konventionen brauche. Der Wandel zu besserem Umweltschutz auf internationaler Ebene gehe sehr langsam und es bestehe die Tendenz, die Verantwortung immer von der eigenen Regierung auf andere Länder zu schieben. Um dieses Problem zu durchbrechen brauche man eine Koalition der Willigen, die kooperieren und auch ohne die Beteiligung anderer Staaten handeln. Zurzeit lässt sich jedoch ein Trend feststellen, nach dem Umweltpolitik immer mehr zu einem Wettbewerb wird, wodurch sich nun auch Länder daran beteiligen, denen es nicht primär um Umweltschutz, sondern um wirtschaftlichen Profit geht. Dies ist einerseits positiv, denn neue Energien können ausgebaut werden. Auch Deutschland beteiligt sich an dieser Wettbewerbsorientierung, Umweltpolitik ist mittlerweile zum Mainstream geworden. Andererseits wird die starke Wettbewerbsorientierung zur Gefahr, wenn man sie als Win-win- Situation sieht, sie sich aber nicht als solche herausstellt. Im Anschluss an dieses Thema kam die Diskussion auf, ob es in Entwicklungs- und Schwellenländern ebenfalls ein Umweltbewusstsein gebe. Diese Frage wurde von Loske mit ja beantwortet, da er eine postmaterialistische Einstellung nicht als Voraussetzung sehe.

Zum Abschluss des Tages hielt Herbert Obinger von der Universität Bremen einen Vortrag darüber, wie man sich als Nachwuchswissenschaftler am besten auf wissenschaftlichen Konferenzen präsentiert. Dieser humoristische Ausflug sollte den jungen Wissenschaftlern einige Tipps vermitteln und ihnen vor allem verdeutlichen, dass sie solche Veranstaltungen nutzen können, um Feedback zu eigenen Arbeiten zu erhalten und Kontakte zu knüpfen.

Der dritte Tag wurde eingeleitet von Annette Töller von der Fernuniversität Hagen und dem Thema Voluntary Approaches in Environmental Policy. Zu diesem Thema wird demnächst ein Sonderheft der German Policy Studies erscheinen. Es lässt sich feststellen, dass zum Thema Umweltpolitik oft deskriptive Fragestellungen entwickelt werden, die eine bestimmte Erkenntnisabsicht haben. Wenige Studien werden durchgeführt, bei denen die Umweltpolitik die abhängige Variable ist. Viele umweltpolitische Fragen können nur mit Blick auf die Institutionen beantwortet werden. Gerade im Umweltbereich sind die Akteure nicht rational, sondern ändern ihre Ziele oft. Zum Beispiel wurden ökonomische Instrumente so lange abgelehnt, bis man ihren Nutzen erkannt hat. Auch gibt es nicht mehr nur weiche Steuerungsinstrumente in der Umweltpolitik, sondern immer mehr Gesetze. So kann es aber sein, dass sich Maßnahmen gegenseitig ausschließen. Deswegen haben sich immer mehr Akteure der freiwilligen Regulierung zugewandt, welche in Deutschland bereits weit verbreitet ist. Sie verliert jedoch im Zuge eines Wandels der Regierungsformen an Bedeutung. Diese Tatsache führte 
im Anschluss an den Vortrag zu einer Diskussion über die Frage, ob es überhaupt sinnvoll sei, zu diesem Thema ein Sonderheft herauszugeben. Hierzu stellte Frau Töller fest, dass trotz der zurückgehenden Bedeutung der freiwilligen Selbstregulierung dieses Phänomen noch nicht hinreichend untersucht worden sei.

Fritz Sager von der Universität Bern stellte die Qualitative Comparative Analysis nach Charles Ragin auf Grundlage eines Beispiels aus seiner eigenen Forschung vor. Ausgangsüberlegung des Ansatzes ist es, eine vermittelnde Position zwischen qualitativer Methode und quantitativer Methode zu finden, so dass mit einer mittleren Zahl von Fällen gearbeitet werden kann. Es wird davon ausgegangen, dass weder das Untersuchen eines Falles, noch die Analyse von Variablen für sich sinnvoll zur Anwendung in den Sozialwissenschaften ist. Beide Ansätze sollen also vereint werden, damit eine komplexe Kausalität festgestellt werden kann, denn ein Ergebnis kann durch verschiedene Voraussetzungen zustande gekommen sein. Bei der Interpretation der Ergebnisse werden deterministische Aussagen gemacht, jedoch ist die Bedingung für Erfolg mit diesem Ansatz, dass die Fallkenntnisse besonders gut sind.

Der letzte Vortrag der diesjährigen Masters Spring Academy wurde von Roland Willner von der Universität Hamburg gehalten, der über Mikropolitik sprach. Es handelt sich hierbei um eine Institutionentheorie, welche Handlungsspielräume in den gegebenen Strukturen betrachtet und feststellt, unter welchen Umständen Interaktionen der Akteure stattfinden. Dabei gibt es zwei verschiedene Perspektiven: die aspektuale und die konzeptuale Perspektive. Es wird davon ausgegangen, dass den Akteuren ein bestimmter Pool an Praktiken zur Verfügung steht, die Handlungsmuster und kollektive Wissensbestände beinhalten. Der Fokus der Untersuchung wird auf die ablaufenden Prozesse und nicht auf die Akteure und Institutionen gerichtet, indem man versucht, diese vergleichbar zu machen. Es wird davon ausgegangen, dass die Handlungsmuster sich durch erneute Anwendung immer wieder reproduzieren, aber auch durch Machtkämpfe bestimmt werden. Durch diese Praktiken wird der Gegensatz von for- mell und informell aufgehoben, denn auch die Prozesse im „Schatten der Hierarchie“ sind wichtig für die Analyse. Allerdings beeinflussen neben vorgegebenen Handlungsmustern und Machtkämpfen auch Kontextfaktoren die Entscheidungen. Bisher wurde der Mikropolitikansatz in den Politikwissenschaften noch wenig genutzt, bietet aber viele Möglichkeiten, zum Beispiel für die Analyse bürokratischer Organisationen oder Regierungsstile.

Insgesamt lässt sich feststellen, dass das Gesamtkonzept einer Verbindung von Lehrkursen für fortgeschrittene Nachwuchswissenschaftler mit der Beteiligung an konkreten Forschungsprojekten und Berichten aus der Praxis nicht nur für die Policy Analyse vielversprechend ist. Der Austausch zwischen Studierenden und Lehrenden aus unterschiedlichen Universitäten war auch in Bremen für alle Beteiligten gewinnbringend. Schade war lediglich, dass angesichts der großen Nachfrage nicht alle Anmeldungen berücksichtigt werden konnten.

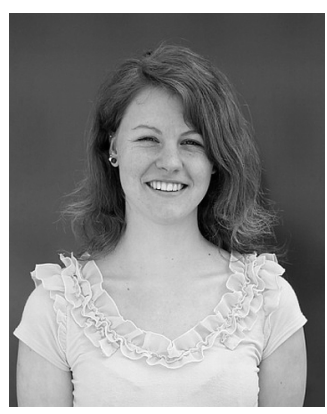

Miriam Düsterhoft ist studentische Hilfskraft am Institut für Sozialwissenschaften der TU Braunschweig mit dem Schwerpunkt Sozial- und Gesundheitspolitik. Email: m.duesterhoeft@tu-braunschweig.de

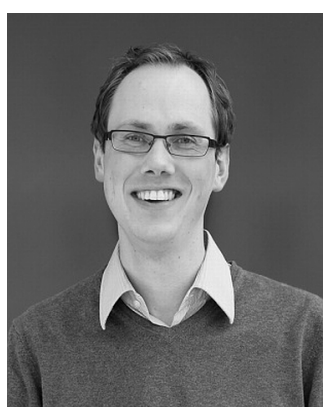

André Völker ist studentische Hilfskraft am Institut für Sozialwissenschaften der TU Braunschweig mit dem Schwerpunkt Sozial- und Gesundheitspolitik. Email: a.voelker@tu-braunschweig.de

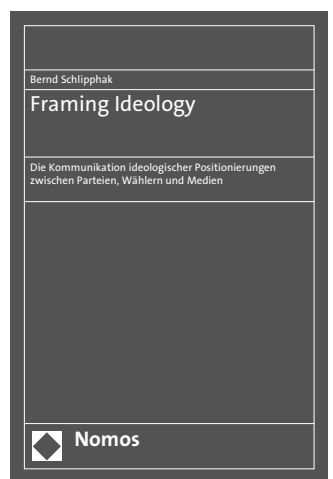

\section{Framing Ideology}

Die Kommunikation ideologischer Positionierungen zwischen Parteien, Wählern und Medien Von Bernd Schlipphak

2011, 301 S., brosch., 49,-€, ISBN 978-3-8329-6301-9

nomos-shop.de/13273

Das Buch führt erstmals auf theoretischer wie empirischer Ebene Ansätze aus Wahlverhaltens-, Parteien- und Medienwirkungsforschung zusammen. Das dafür entwickelte Modell des Framing wird anhand der Effekte des Frames „Ideologie“ mit quantitativen Primär- und Sekundärdaten am Fallbeispiel der ideologischen Verortung der SPD zwischen 1998 und 2005 überprüft.

Bitte bestellen Sie im Buchhandel oder versandkostenfrei unter $\downarrow$ www.nomos-shop.de

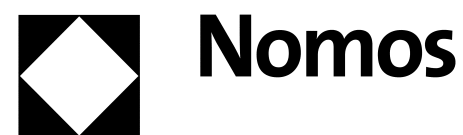

